Harris Lauren (Orcid ID: 0000-0003-0903-8278)

\title{
Clinicopathologic Features of Lingual Canine T-Zone Lymphoma
}

Lauren J. Harris ${ }^{1}$, Emily D. Rout ${ }^{1}$, Kelly L. Hughes ${ }^{1}$, Julia D. Labadie ${ }^{1}$, Brendan Boostrom ${ }^{2}$, Janna A. Yoshimoto $^{1}$, Claire M. Cannon ${ }^{3}$, Paul R. Avery ${ }^{1}$, E.J. Ehrhart ${ }^{1}$, Anne C. Avery ${ }^{1}$

${ }^{1}$ Department of Microbiology, Immunology and Pathology, College of Veterinary Medicine and Biomedical Sciences, Colorado State University, Fort Collins, CO

${ }^{2}$ Clinical Sciences Department, College of Veterinary Medicine and Biomedical Sciences, Colorado State University, Fort Collins, CO

${ }^{3}$ Department of Veterinary Clinical Sciences, College of Veterinary Medicine, University of Minnesota, St. Paul, MN

This is the author manuscript accepted for publication and has undergone full peer review but has not been through the copyediting, typesetting, pagination and proofreading process, which may lead to differences between this version and the Version of Record. Please cite this article as doi: $10.1111 /$ vco.12322

This article is protected by copyright. All rights reserved. 

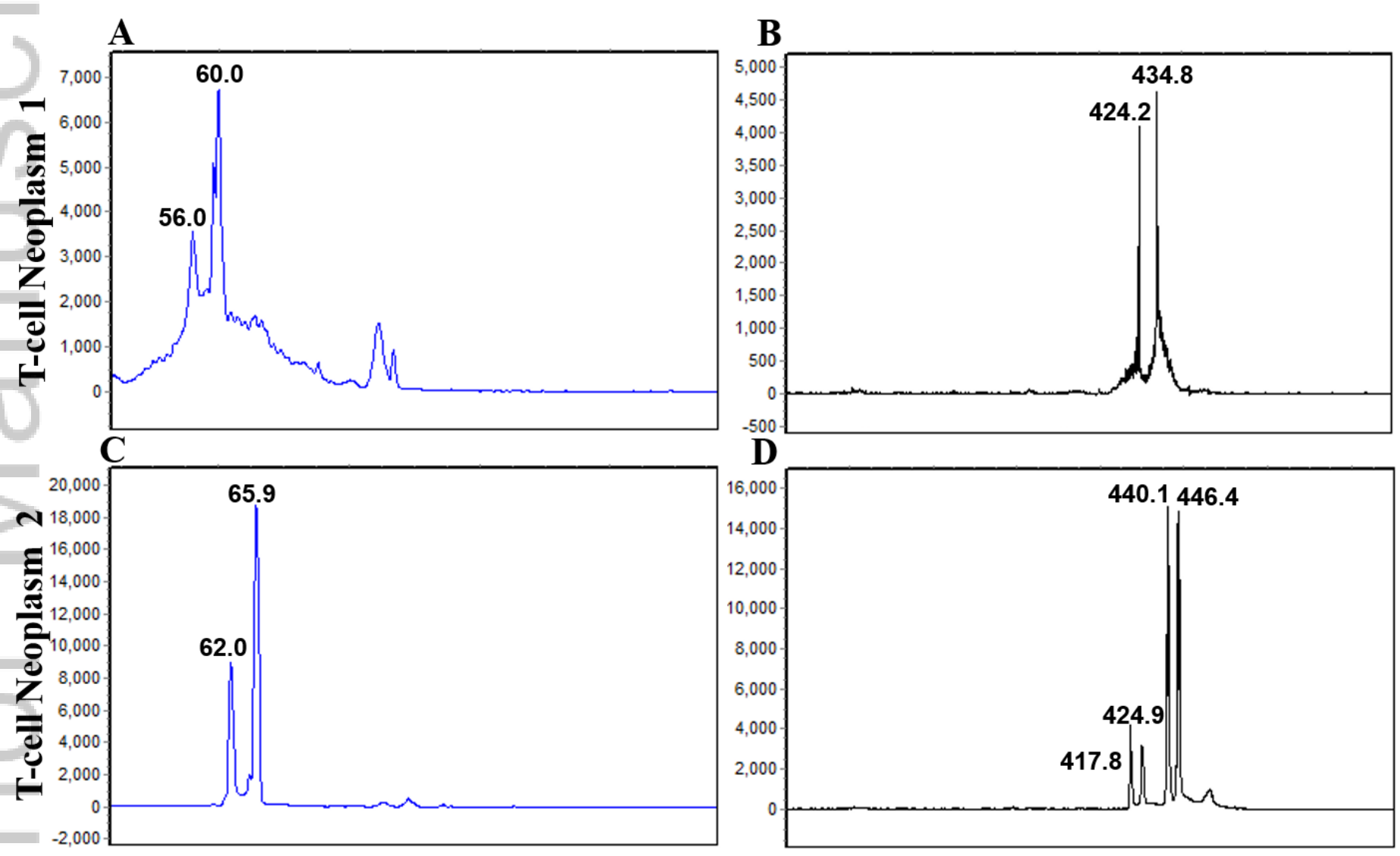

Figure 5.tif

This article is protected by copyright. All rights reserved. 
Figure 1. Representative gross lesions on the tongues of four different dogs (A-D). The dorsal ventral and/or lateral aspects of the tongue are expanded by multifocal to coalescing raised red nodular masses.

Figure 2. Cytologic findings from paired peripheral lymph node (A,B) and tongue masses (C,D) from two dogs (case 11 and case 1) with TZL. Wright-Giemsa, x60 objective. Lymphocytes are intermediate in size with a small round nucleus, coarse chromatin and expanded pale blue cytoplasm, often forming a "mirror-handle" appearance (arrows). There are few small welldifferentiated lymphocytes admixed with neoplastic cells (arrowheads).

Figure 3. Histologic features of lingual TZL from two representative dogs. Expansile nodules of neoplastic round cells expand the superficial submucosa (A, C), H\&E, x4 objective. Neoplastic cells are nonepitheliotropic. Cells are arranged in vague packets overlying a fine fibrovascular stroma, have expanded pale eosinophilic cytoplasm and round to indented nuclei with condensed homogeneous chromatin and inconspicuous nucleoli (B,D), H\&E, x40 objective.

Figure 4. Immunohistochemistry profile of lingual TZL. Neoplastic cells exhibit strong CD3 immunoreactivity (A,B), x10;x40 objectives respectively. Neoplastic cells do not exhibit positive immunoreactivity to the Pax5 antibody. There are few scattered Pax5 positive B cells at the periphery of the neoplastic nodules (C,D), x10;x40 objectives respectively. Neoplastic cells 
diffusely do not demonstrate immunoreactivity to the Muml antibody, a plasma cell marker (E,F) x10;x40 objectives respectively.

Figure 5. PARR assay of case 9 demonstrating two different clonal TCR rearrangements supportive of a diagnosis of two separate T-cell neoplasms. The low molecular weight PCR products in blue $(\mathrm{A}, \mathrm{C})$ are amplified with $\mathrm{V}$ gamma 7 primers and the larger products in black (B,D) are amplified with V gamma 3 primers. The first T-cell neoplasm $(\mathrm{A}, \mathrm{B})$ and second T-cell neoplasm (C,D) both exhibit clonal rearrangements with different sized molecular weight products. 

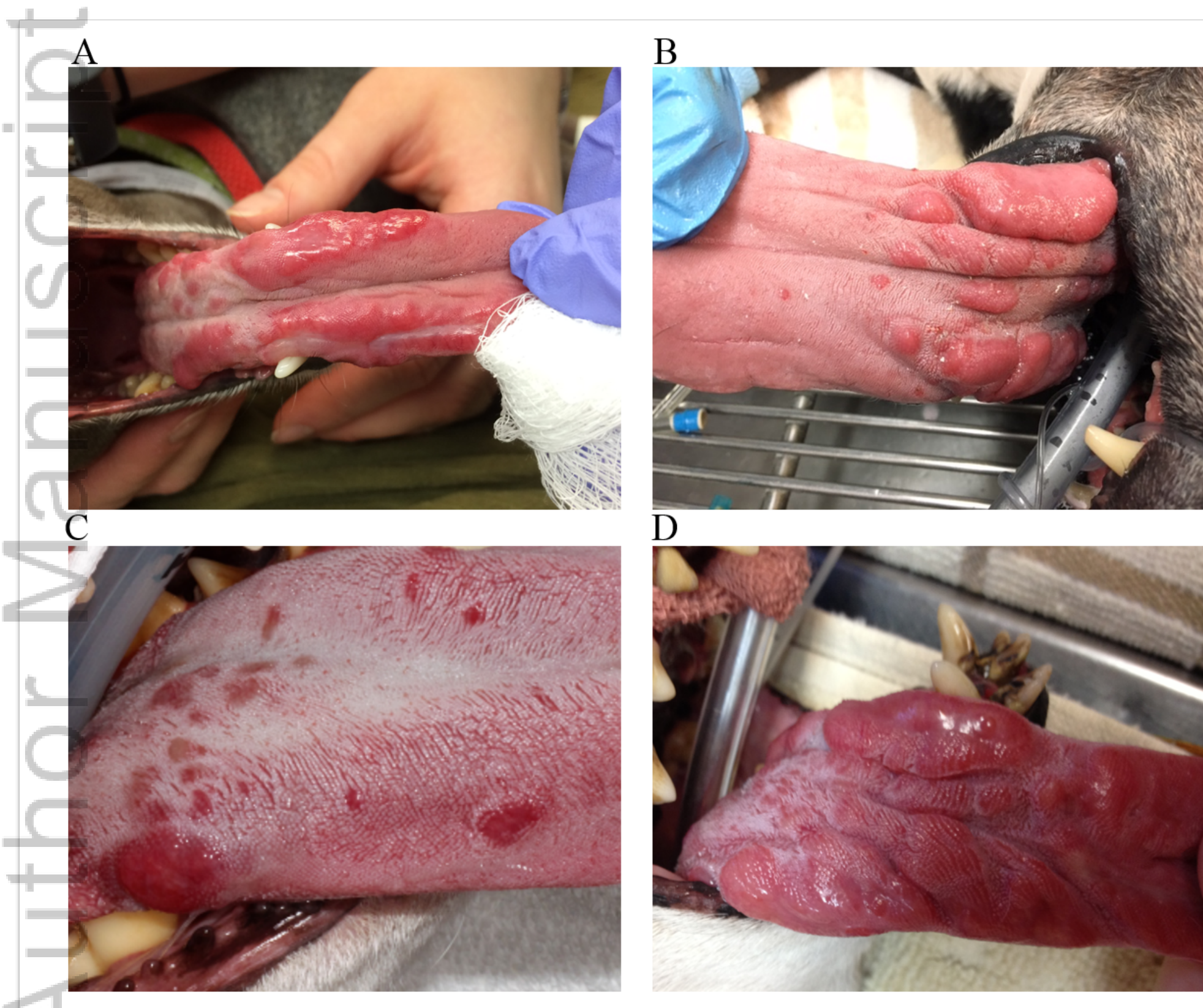

Figure_1.tif

This article is protected by copyright. All rights reserved. 


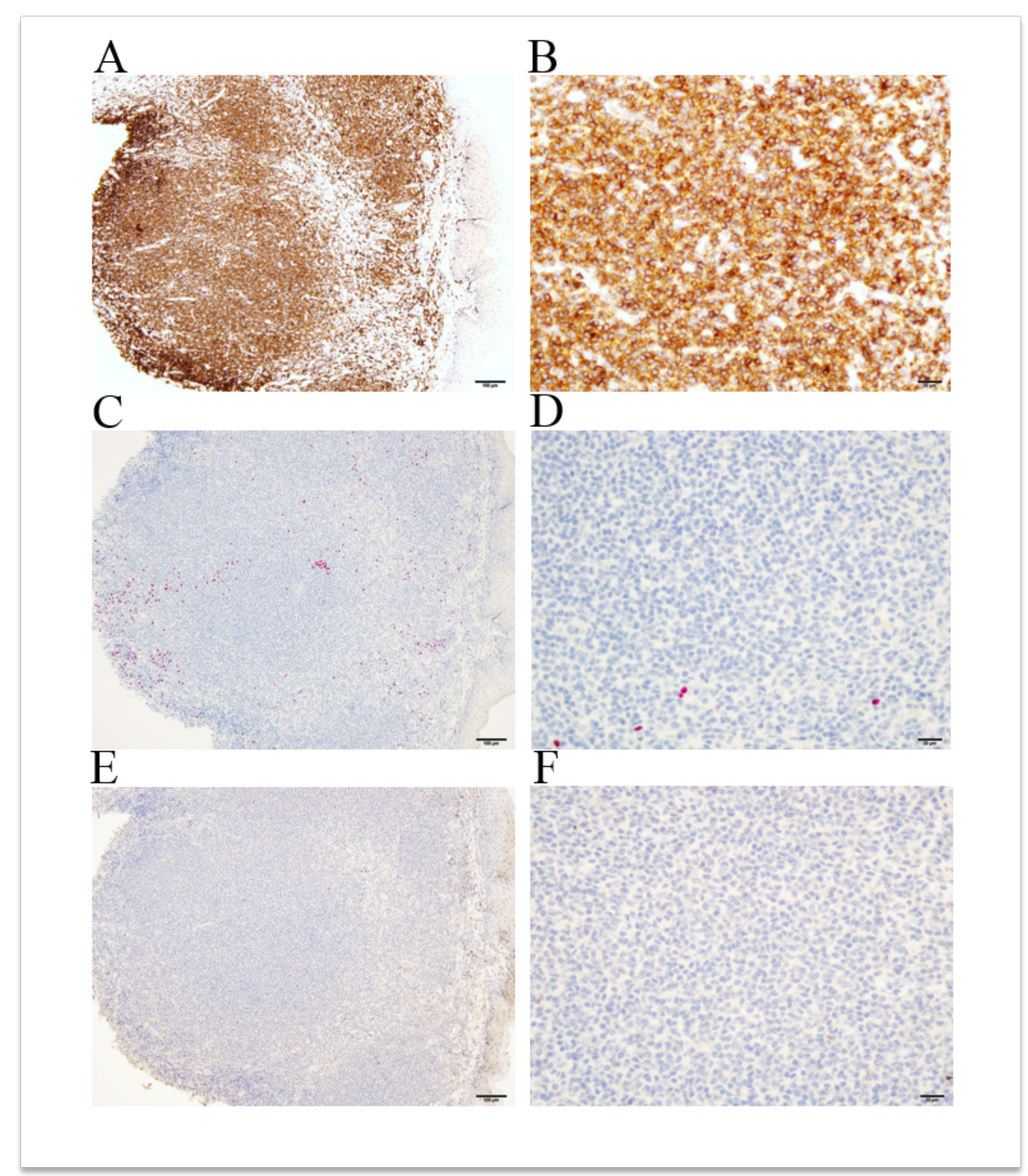

Figure_4.tif

This article is protected by copyright. All rights reserved. 


\begin{abstract}
:
Canine T-zone lymphoma (TZL) is a subtype of T-cell lymphoma characterized by unique histologic pattern and cytomorphology, immunophenotypic loss of CD45 expression, and an indolent clinical behavior. Dogs with TZL typically present with one or more enlarged lymph nodes and/or lymphocytosis. We describe a novel extranodal presentation of TZL involving the tongue. Twelve dogs with tongue masses were diagnosed with lingual TZL based on a variable combination of immunophenotyping via flow cytometry, cytology, histopathology, immunohistochemistry, and/or PCR for antigen receptor rearrangement (PARR) assay. Eleven dogs exhibited concurrent lymphocytosis and/or lymph node enlargement. Three cases were initially diagnosed as plasma cell tumors based on histology alone, thereby revealing a potential diagnostic challenge. Seven dogs achieved clinical remission and four achieved stable disease following variable treatment, consistent with the indolent nature of typical TZL involving the lymph nodes and peripheral blood. In one case the TZL resulted in progressive disease and failure to respond to treatment. In this case, the TZL exhibited histologic features of a higher grade neoplasm. This case series highlights a unique presentation of TZL and identifies a new differential diagnosis for lingual neoplasia. In this study we characterize the clinical presentation, diagnostic features, and patient outcomes of twelve dogs with lingual TZL.
\end{abstract}

KEYWORDS: Canine; Tongue; Neoplasia; Lymphoma; T-Zone 


\section{INTRODUCTION:}

Canine T-cell lymphomas encompass a broad spectrum of diseases with diverse biologic behaviors and responses to treatment. Among this group is canine T-zone lymphoma (TZL) which is characterized by an indolent clinical course in cases involving lymph nodes and peripheral blood with reported median survival times ranging from 21.2 to 33.5 months. ${ }^{1-3} \mathrm{TZL}$ is estimated to be fairly common, comprising $3-12 \%{ }^{4,5}$ of all canine lymphomas and up to $60 \%$ of indolent canine lymphomas. ${ }^{1}$ Affected dogs typically present with one or more enlarged lymph nodes and/or lymphocytosis, ${ }^{2,4,6,7}$ often identified as an incidental finding with no associated change in clinical behavior ${ }^{4}$ Circulating neoplastic T-zone cells can be identified in dogs with and without clinical lymphocytosis ${ }^{2}$ and the presence of circulating T-zone cells within the peripheral blood is not associated with a worse prognosis. ${ }^{1}$

Reports of TZL involving sites other than peripheral lymph nodes and blood are limited. One report describes indolent non-epitheliotropic cutaneous T-cell lymphoma in eight dogs with complete or partial loss of the CD45 antigen. ${ }^{8}$ T-zone lymphoma cells reliably exhibit loss of CD45 ${ }^{2,9}$ suggesting that these eight cases may be consistent with TZL. Dogs in this report presented with erythematous, scaly and alopecic macules, patches or plaques in the axillae, inguinal region, thorax, abdomen, flank, thighs, legs, head and neck. Clinically these dogs exhibited a prolonged quiescent stage and slow progression of disease ${ }^{8}$ consistent with the indolent behavior of TZL.

We report a novel presentation of TZL involving the tongue of twelve dogs. Samples from affected dogs were submitted to the Clinical Immunology Laboratory and/or the Veterinary Diagnostic Laboratories at Colorado State University between 2006 and 2016 and TZL was diagnosed based on a variable combination of cytology, histopathology, immunohistochemistry, immunophenotyping via flow cytometry, and PCR for antigen receptor rearrangement (PARR) assay (Table 1). The goal of this study is to identify the presenting characteristics, diagnostic features, and clinical outcomes of this newly identified entity. 


\section{METHODS:}

\section{CASE SELECTION:}

The majority of cases were identified through the Colorado State University Clinical Immunology (CSU-CI) laboratory. The CSU-CI database was searched for dogs where flow cytometry had been performed on a sample from a patient for which either "tongue" or "oral" was listed as an affected site. All cases with immunophenotypic loss of CD45 were selected for further investigation. Additional cases were identified through the CSU-Veterinary Diagnostic Laboratory by searching for oral lymphoma and then identifying lesions with morphology consistent with TZL. Selected cases were further evaluated by variable combination of flow cytometry, cytology, histopathology, immunohistochemistry and/or PARR assay (Table 1), resulting in consensus diagnosis of TZL. Evaluation of all diagnostic modalities in all cases was limited by sample availability. Medical records were obtained from the Colorado State University Veterinary Teaching Hospital (CSU-VTH) $(n=3)$ or from referring veterinarians for patients not treated at the CSU-VTH $(n=9)$. Gross descriptions and digital images $(n=5)$ of lesions at initial presentation were obtained from medical records. Physical examination records and $\mathrm{CBC}$ at time of presentation, or the most recent record after initial presentation, were evaluated for changes in lymph node size and lymphocytosis. Treatment protocols and clinical responses were obtained from hospital records or via direct communication with the primary veterinarian in cases where this information was not present in the record.

\section{FLOW CYTOMETRY:}

Flow cytometry was performed on the tongue mass and lymph node $(n=4)$, lymph node alone $(n=1)$, or peripheral blood alone $(n=6)$ as previously described. ${ }^{2}$ The use of peripheral blood or lymph node alone in the diagnosis of TZL has been validated in a previous study ${ }^{2}$ in which all cases with phenotypic loss of CD45 antigen expression were confirmed as TZL by 
histopathology. In this report, all cases with confirmed histologic diagnoses of TZL in the lymph node had detectable circulating neoplastic (TZL) cells, even those patients without overt lymphocytosis. Diagnosis of the tongue lesions was based on characteristic histologic pattern or cytologic morphology and in some cases was supported by immunophenotyping of peripheral blood, lymph node, or tongue mass.

\section{CLONALITY TESTING:}

The presence of a clonally expanded lymphocyte population was detected by PARR (PCR for antigen receptor rearrangement) assay on the tongue mass $(n=1)$, lymph node $(n=3)$, or peripheral blood ( $\mathrm{n}=5)$ as previously described. ${ }^{10,11}$ A reaction was considered clonally positive (T-cell receptor rearrangement) if one or more discrete PCR products dominated with distinct peak in the electropherogram view in which the height was three times the size of the base. If multiple peaks were seen in a Gaussian distribution, the reaction was considered polyclonal (negative for clonality). The reaction was considered equivocal when the peak did not reach objective criteria but was still subjectively suspicious for clonality.

\section{CYTOLOGY AND HISTOLOGY:}

Lymph node and tongue lesion fine-needle aspirates were air-dried and stained with Giemsabased stains. Five $\mu \mathrm{m}$ paraffin-embedded histologic preparations stained with hematoxylin and eosin (H\&E) were obtained from the Colorado State University Diagnostic Laboratory, Antech Diagnostics, or VDx Veterinary Diagnostics. Cytology slides were reviewed by a single clinical pathologist (PRA) and resident (EDR) and histology slides were reviewed by two anatomic pathologists $(\mathrm{EJE}, \mathrm{KLH})$ and resident $(\mathrm{LJH})$. In cases where cytology $(\mathrm{n}=6)$ or histology $(\mathrm{n}=2)$ were initially performed but tissue was no longer available for re-evaluation, the initial pathology report was reviewed. 


\section{IMMUNOHISTOCHEMISTRY:}

Heat-induced epitope retrieval was performed on a Leica Bond-Max or Leica Bond III IHC stainer using Bond Epitope Retrieval Solution 2 (Bond Epitope Retrieval Solution 2, Leica Biosystems Newcastle Ltd, Newcastle Upon Tyne, United Kingdom) for 30 minutes. Neoplastic lymphocytes were immunophenotyped as T-cells using monoclonal mouse anti-human CD3 (LN10, Leica Biosystems Newcastle Ltd, Newcastle Upon Tyne, United Kingdom). Neoplastic lymphocytes were additionally evaluated for B-cell immunophenotype using monoclonal mouse anti-human B-cell-specific activator protein PAX-5 (DAK-Pax5, Dako North America Inc., Carpinteria, CA) or with an alternate B-lymphocyte antigen using monoclonal mouse anti-human CD79a (HM57, Dako North America Inc., Carpinteria, CA). In one case neoplastic cells were also evaluated for plasma cell immunophenotype using the monoclonal mouse anti-human MUM1 protein, (Mum1p, Dako, North America Inc., Carpinteria, CA). Labeling was performed on an automated staining platform (Bond-Max, Leica Biosystems Newcastle Ltd, Newcastle Upon Tyne, United Kingdom). Fast Red (Fast Red Substrate System, Dako North America Inc., Carpinteria, CA) or 3,3'-Diaminobenzidine (DAB) (liquid DAB+ Substrate, Dako North America Inc., Carpinteria, CA) were used as chromogens and slides were counterstained with hematoxylin. Immunoreactions were visualized using commercial detection systems (Bond Polymer Refine detection system, Leica Biosystems Newcastle Ltd, Newcastle Upon Tyne, United Kingdom; Bond Polymer Refine Red detection system, Leica Biosystems Newcastle Ltd, Newcastle Upon Tyne, United Kingdom). In all cases, normal and reactive canine lymph node tissues incubated with primary antibodies were used as positive immunohistochemical controls. Negative controls were incubated in diluent consisting of Tris-buffered saline with carrier protein and homologous nonimmune sera. All sequential steps of the immunostaining procedure were performed on negative controls following incubation.

\section{OUTCOME ASSESSMENT:}


Response to treatment was determined based on reported changes in size of the tongue lesions in medical records or through direct communication with the primary veterinarian. Due to the difficulty in repeated measurements in the oral cavity, multifocal to coalescing pattern of the tongue lesions, and retrospective nature of the study a modified RECIST criteria was utilized for evaluation of disease course. Complete remission (CR) was defined as complete resolution of the tongue lesions. Stable disease (SD) was defined as static or slightly improved tongue lesions. Partial remission (PR) was defined as smaller but not completely resolved tongue lesions. Progressive disease (PD) was defined as enlarged tongue lesions. Follow-up time was calculated from the time of initial diagnosis to the last recorded evaluation of the tongue lesions.

\section{RESULTS:}

\section{PATIENT CHARACTERISTICS:}

Samples from 12 dogs submitted to the Colorado State University Clinical Immunology Laboratory and/or Veterinary Diagnostic Laboratories from 2006-2016 for evaluation of lesions on the tongue met inclusion criteria. Diagnosis of tongue TZL was based on a variable combination of immunophenotyping via flow cytometry, cytology, histology, and immunohistochemistry (Table 1). Patient characteristics and clinical presentation are summarized in Table 2. The median age was 9.5 years with a range of 7-12 years. Golden Retrievers were the most frequently represented breed $(n=4,33 \%)$, consistent with the previously reported breed predilection of TZL with $40 \%$ of all affected dogs being Golden Retrievers. ${ }^{2}$

The majority of the dogs were asymptomatic $(n=9)$ at the time of initial presentation. Primary care veterinarians identified lesions during a dental procedure $(n=6)$ or during routine physical examination $(n=3)$. Fewer $\operatorname{dogs}(n=3)$ presented with symptoms associated with the oropharynx including drooling excessively $(n=1)$, increased respiratory noise $(n=1)$, and change in bark $(n=2)$. One of these three dogs additionally presented with trouble eating, drinking, and breathing due to the space occupying tongue mass. 
The gross appearance of lesions on the tongue was consistent throughout the cases. In all cases the dorsal, ventral, and/or lateral aspects of the tongue were thickened by multifocal to coalescing, raised, red, nodular masses (Figure 1). Few cases $(n=2)$ involved superficial mucosal ulceration on gross and/or histologic evaluation.

The majority of dogs presented with concurrent lymphadenomegaly and/or lymphocytosis (Table 2). The most commonly affected lymph nodes included mandibular only $(n=3)$ or mandibular, prescapular, and popliteal $(n=3)$. In one case the mandibular, popliteal, and iliac lymph nodes were enlarged and in one additional case only the laryngeal lymph nodes were enlarged. Seven dogs presented with lymphocytosis, based on bloodwork performed closest to the time of presentation, with a median lymphocyte count of 9,454 cells/uL and range of 7,11016,100 cells/uL.

\section{IMMUNOPHENOTYPING AND ASSESSMENT OF CLONALITY:}

Flow cytometry was performed on 11 of 12 cases and was carried out on the tongue mass and lymph node $(n=4)$, lymph node alone $(n=1)$, or peripheral blood alone $(n=6)$. In cases where multiple sites were evaluated the phenotype was consistent across samples. All of the examined cases had an expansion of T-cells that had lost expression of the pan-leukocyte marker CD45 (CD45-), which is diagnostic for TZL. ${ }^{2}$ Nine (81\%) of the cases involved CD8 T-cells, 1 (9\%) expressed CD4, and 1 (9\%) did not express CD4 or CD8 (CD4-CD8-). A greater percentage of cases in this series are CD8+ when compared to the overall population of reported T-zone cases in which $33-45 \%$ of cases are CD8+, $15-16 \%$ of cases are CD4+, and $40-49 \%$ of cases are CD4CD8-. ${ }^{2}$

PCR for antigen receptor rearrangements (PARR) assay was performed in 9 cases. Samples were obtained from blood $(n=5)$, lymph node $(n=3)$, or the tongue mass $(n=1)$. Eight out of 9 cases exhibited a clonal T-cell receptor rearrangement, consistent with TZL. One dog (case 4) had a negative PARR result which is likely a false negative result, as $15 \%$ of confirmed 
malignancies will be negative via the PARR assay (data not published). Diagnosis of TZL in this case was supported by histology of the tongue mass, cytology of the lymph node, flow cytometry of the blood, clinical lymphocytosis, enlarged submandibular lymph nodes, and resolution of the tongue lesions and lymphocytosis following treatment with chlorambucil and prednisone.

\section{CYTOLOGIC FEATURES:}

Fine needle aspiration was performed on the tongue mass $(n=3)$ and/or lymph node $(n=7)$ and the cytologic preparation or initial cytology report in cases where samples were no longer available $(\mathrm{n}=6)$ was reviewed by a clinical pathologist (PRA) and resident (EDR). In all examined cases there was an expansion of small to intermediate sized lymphocytes with consistent cellular morphology (Figure 2). Lymphocytes were intermediate in size (15-20 um) with a small round nucleus (10-12 um), coarse nuclear chromatin and rarely one small faint nucleolus. Cells had moderately expanded pale blue cytoplasm, which extended asymmetrically, forming a wide-base "mirror-handle" appearance. In the tongue mass samples, these lymphocytes were the predominant cell population, with rare small well-differentiated lymphocytes in the background. In the lymph node aspirates, the neoplastic lymphocytes accounted for $30-95 \%$ of the cell population.

One of the three cytologic samples from the tongue was no longer available for examination but the report described a consistent cellular morphology. Six of the 7 lymph node cytology reports specifically described an expansion of small to intermediate sized lymphocytes with 'mirror-handle' shaped cytoplasm, consistent with the previously described morphology of T-zone lymphocytes. ${ }^{7,12}$ One (case 4 ) of the seven lymph node aspirates was diagnosed as atypical lymphoid hyperplasia or possible intermediate-cell lymphoma with an expanded intermediate lymphocyte population (80-90\% of the population). This sample was no longer available for review but could be consistent with T-zone morphology since TZL is composed of intermediate sized cells and it is common for T zone lymphomas to be described cytologically as 
"lymphoid hyperplasia" or "atypical lymphoid hyperplasia", with a subsequent diagnosis by flow cytometry or histopathology of T-zone lymphoma (AA unpublished observations).

\section{HISTOPATHOLOGIC AND IMMUNOHISTOLOGIC FEATURES:}

Histology of the tongue masses was available for evaluation in 9 of the 12 cases and histopathologic features were conserved across all examined cases. Histopathologic features (Figure 3) included expansile nodules, densely cellular sheets, and faint packets of monomorphic neoplastic round cells overlying a fine fibrovascular stroma. Neoplastic cells expanded the superficial submucosa, were separated from the epidermis by a thin band of connective tissue, and did not show evidence of epitheliotropism. Cells were small to intermediate in size with nuclei that were 1-1.5 times the size of a red blood cell and increased amounts of pale amphiphilic to eosinophilic cytoplasm. Nuclei were oval and frequently indented with homogeneous, condensed chromatin and indistinct nucleoli.

The mitotic rate was variable, ranging from $2-75$ per ten 400x fields. The majority of cases had less than 10 mitoses per ten 400x fields ( 6 out of 9); fewer cases ( 2 out of 9 ) had between 10-20 mitoses per ten 400x fields; and one case (case 12) had a much higher mitotic rate of 75 mitoses per ten $400 x$ fields. In this case there were multifocal regions of necrosis that effaced approximately $30 \%$ of the examined cross sectional tumor area, a feature not evident in the other evaluated cases. Occasionally admixed with neoplastic cells were low numbers of eosinophils, neutrophils, and rare mast cells.

The histopathology reports for two additional cases in which tissue was no longer available were reviewed. In both cases non-epitheliotropic round cell neoplasms of consistent morphology and low mitotic rates were described. Diagnosis of TZL in both of these cases was supported by the CD45 negative immunophenotype via flow cytometry. 
Three cases were initially diagnosed as possible plasma cell tumors (plasmacytoma) based on histologic pattern alone. In all three cases, ancillary testing (immunohistochemistry and/or flow cytometry) subsequently revised the diagnosis to TZL.

Immunohistochemistry to characterize T-cell versus B-cell origin was performed on 9 of the 11 total cases with histopathology available (Figure 4). Neoplastic cells diffusely demonstrated positive immunoreactivity to the CD3 antibody, a marker of T-cells. Low numbers of B-cells, identified with PAX5 or CD79a antibodies, were clustered and compressed at the periphery of the neoplastic nodules. The B-cell population is consistent with compressed follicles similar to those present in TZL in the lymph node. ${ }^{2}$ In one case, MUM1 immunoreactivity was evaluated and neoplastic cells were negative for the plasma cell marker.

\section{TREATMENT AND OUTCOME:}

All cases $(n=12)$ were treated and a variety of treatment protocols were initiated ranging from surgical excision to multi-agent chemotherapy to palliative radiation. Clinical therapy and response to treatment is summarized in Table 3. Due to the low sample size, variety of treatments, and retrospective nature of this study correlation between treatments and outcome was not attempted.

The majority of dogs (11 of 12) achieved complete remission or stable disease. Dogs reaching stable disease were diagnosed 27-149 days from the time of manuscript preparation and therefore assessment of response to treatment is limited by short follow-up time. Two of these cases (case 10, case 11) were treated with amoxicillin-clavulanic acid and the tongue lesions were static to slightly smaller following treatment. One dog (case 8) began chlorambucil following mild progression of the lesions and had stable to slightly improved disease 90 days after starting treatment. The fourth dog (case 9) was monitored for approximately four months with eventual increase in size of the tongue lesions. Palliative radiation therapy to the tongue was subsequently pursued in this case. Following completion of four out of four radiation treatments the lesions 
were stable to improved. Approximately 1.5 months following completion of radiation therapy, this dog presented with pleural effusion and was diagnosed with a second T-cell neoplasm. This neoplastic population had a different immunophenotype (CD3+CD5+CD4-CD8-CD45+), indicative of an additional T-cell neoplasm as opposed to progression of the TZL. The presence of a second T-cell neoplasm was further confirmed with PARR assay (Figure 5).

Three of the patients who achieved a complete remission had relapse of their tongue lesions 140-750 days following initiation of treatment and all three patients achieved a second complete remission. In two cases a second remission was achieved after rescue therapy with prednisolone and/or chlorambucil (case 2 and 3) and in one case the lesions spontaneously resolved (case 6).

Ten of twelve dogs were still alive at publication (27-893 days post-diagnosis) and two dogs have been euthanized. Median overall survival time could not be calculated because only two dogs died during the study period (14 and 379 days). One dog (case 4) was euthanized for a non-healing wound on the hind limb 13 months after TZL diagnosis. Biopsy and histopathology of the non-healing wound was consistent with an acral lick granuloma with no evidence of neoplasia. The second dog (case 12) was euthanized due to the space occupying effect of TZL. In this case, the owner noticed the tongue mass two weeks prior to euthanasia and it was reported to rapidly increase in size. The dog was treated with a one-week course of prednisone with no clinical improvement. The histologic features of this dog's tumor also appear more aggressive (mitotic rate of 75 mitoses per ten 400x fields and multifocal areas of tumor necrosis). The biologic behavior and histopathologic features of this neoplasm are distinctly different from the other examined cases and may represent a more biologically aggressive presentation of TZL.

\section{DISCUSSION:}

We identify a unique presentation of canine TZL involving the tongue. Cases of lingual TZL demonstrate $(\mathrm{CD} 3+) \mathrm{T}$-cell origin and immunophenotypic loss of CD45 expression by flow 
cytometry. Histologic pattern and cytomorphology are consistent with that of the more commonly described nodal presentation of TZL. ${ }^{2,4}$ The indolent clinical course of typical TZL is largely conserved with nearly all of the dogs in this series achieving clinical remission or stable disease. Because the majority of dogs (10 of 12) in this study are still alive and several were recently diagnosed, further evaluation of the biologic behavior of this entity will be dependent on continued patient follow-up.

In one case the TZL demonstrated a more aggressive biologic behavior characterized by rapid clinical onset and progression, lack of response to a one-week course of steroid therapy, and euthanasia secondary to the space-occupying lingual mass. The histologic features of this neoplasm were also suggestive of a high grade neoplasm with a high mitotic rate and multifocal regions of tumor necrosis. The mitotic rates in other cases were also higher than previously reported in nodal TZL. TZL is typically characterized by few to no mitotic figures. ${ }^{2,4}$ In contrast, the mitotic index in this series (excluding the atypical previously described case) ranged from 220 mitoses per ten 400x fields. Exploration of the relationship between mitotic rate and prognosis was not attempted in the study due to the small sample size and limited follow-up interval in several cases.

Correlation between treatment and outcome were also not evaluated due to the limited population and retrospective nature of the study. The majority of dogs in the study achieved clinical remission or stable disease following a variety of treatment protocols. Interestingly, in a previous study of nodal TZL, systemic treatment did not influence clinical outcome. ${ }^{1}$ While conclusions about response to treatment in this study cannot be made due to lack of appropriate control cases, we may postulate that the higher mitotic rate observed within this neoplastic population as compared to nodal TZL may result in a greater susceptibility to systemic therapy. Furthermore, the location of the lesions within the oral cavity can result in associated oropharyngeal symptoms including difficulty eating, drinking, and breathing and therefore may justify systemic treatment. 
The majority of dogs (11 out of 12) presented with concurrent lymphadenomegaly and/or lymphocytosis. Lymphocytosis and lymphadenomegaly are interpreted as findings consistent with T-zone disease ${ }^{1,2}$ rather than indications of advanced stage disease. This conclusion is supported by the finding that in TZL, circulating T-zone cells are present in dogs with and without clinical lymphocytosis ${ }^{2}$ and the presence of these cells in circulation is not associated with a worse prognosis. ${ }^{1}$

The predilection of the tongue in these cases is interesting although the pathogenesis leading to this tissue specificity is not well understood. The presence of low numbers of Pax 5 or CD79a positive B-cells in multiple histologic sections suggests that these lesions may represent neoplastic transformation of mucosa-associated lymphoid tissue (MALT) or infiltration of TZL to a stimulated MALT follicle. MALT in the tongue of dogs is poorly understood. Normal lingual lymphoid tissue has been reported in other domestic animals (ruminants and equine) ${ }^{13}$ and there have been few reports of MALT lymphoma in the tongue of humans. ${ }^{14-17}$ Further investigation into the homing of T-cells to the tongue and characterization of MALT in the tongue of dogs would facilitate a greater understanding of the pathogenesis underlying the tissue specific distribution of this entity.

The characterization of a newly identified lingual presentation of TZL is valuable for pathologists and clinicians to aid in correct diagnosis and appropriate clinical management of lingual masses. In previous surveys of lingual neoplasia in dogs, malignant (epitheliotropic or nonspecified) lymphoma has been reported to comprise $2-7 \%$ of all tongue tumors. ${ }^{18,19}$ Other reported round cell tumors in the tongue included plasma cell tumors (10-12\%) and granular cell tumors (6-10\%). Interestingly, in our study three of the eleven cases evaluated histologically were initially diagnosed as possible plasma cell tumors based on histologic morphology alone. Shared histologic features between plasma cell tumors and T-zone lymphomas include discrete round cell populations with lack of epitheliotropism, vague packeting, and expanded pale staining cytoplasm. Thereby, this entity may present a potential diagnostic challenge, emphasizing the 
importance of ancillary diagnostics including immunohistochemistry and immunophenotyping via flow cytometry to achieve an accurate diagnosis.

Accurate diagnosis of lingual TZL can provide insight into clinical interpretation of associated lymphocytosis and lymphadenopathy. Furthermore, immunophenotyping via flow cytometry can aid in the differentiation between indolent TZL and a more aggressive T-cell neoplasm, which may require more intensive therapy and carry a worse long-term prognosis. Clinical detection of additional cases of lingual TZL and continued case follow-up will facilitate a better understanding of the clinical incidence, response to treatment, and biologic behavior of this entity.

\section{REFERENCES}

1. Flood-Knapik KE, Durham AC, Gregor TP, Sánchez MD, Durney ME, Sorenmo KU. Clinical, histopathological and immunohistochemical characterization of canine indolent lymphoma. Vet Comp Oncol. 2013;11(4):272-286. doi:10.1111/j.14765829.2011.00317.x.

2. Seelig DM, Avery P, Webb T, et al. Canine t-zone lymphoma: Unique immunophenotypic features, outcome, and population characteristics. $J$ Vet Intern Med. 2014;28(3):878-886. doi:10.1111/jvim.12343.

3. Martini V, Marconato L, Poggi A, et al. Canine small clear cell/T-zone lymphoma: clinical presentation and outcome in a retrospective case series. Vet Comp Oncol. 2015;14(S1):117-125. doi:10.1111/vco.12155.

4. Valli VE, San Myint M, Barthel A, et al. Classification of canine malignant lymphomas according to the World Health Organization criteria. Vet Pathol. 2011;48(1):198-211. doi:10.1177/0300985810379428.

5. Ponce F, Marchal T, Magnol JP, et al. A morphological study of 608 cases of canine malignant lymphoma in France with a focus on comparative similarities between canine 
and human lymphoma morphology. Vet Pathol. 2010;47(3):414-433. doi:10.1177/0300985810363902.

6. Valli VE, Vernau W, de Lorimier L-P, Graham PS, Moore PF. Canine indolent nodular lymphoma. Vet Pathol. 2006;43(3):241-256. doi:10.1354/vp.43-3-241.

7. Mizutani N, Goto-Koshino Y, Takahashi M, Uchida K, Tsujimoto H. Clinical and histopathological evaluation of 16 dogs with T-zone lymphoma. J Vet Med Sci. 2016;78(8):1237-1244. doi:10.1292/jvms.15-0688.

8. Affolter VK, Gross TL, Moore PF. Indolent cutaneous T-cell lymphoma presenting as cutaneous lymphocytosis in dogs. Vet Dermatol. 2009;20(5-6):577-585. doi:10.1111/j.1365-3164.2009.00833.x.

9. Martini V, Poggi A, Riondato F, Gelain ME, Aresu L, Comazzi S. Flow-cytometric detection of phenotypic aberrancies in canine small clear cell lymphoma. Vet Comp Oncol. 2013;13(3):281-287. doi:10.1111/vco.12043.

10. Burnett RC, Vernau W, Modiano JF, Olver CS, Moore PF, Avery AC. Diagnosis of canine lymphoid neoplasia using clonal rearrangements of antigen receptor genes. Vet Pathol. 2003;40:32-41. doi:10.1354/vp.40.1.32.

11. Hughes KL, Labadie JD, Yoshimoto JA, Dossey JJ, Burnett RC, Avery AC. Increased frequency of CD45 negative T cells (T zone cells) in older Golden retriever dogs. Submitt to Vet Comp Oncol. 2016.

12. Seelig D, Avery A, Ehrhart E, Linden M. The Comparative Diagnostic Features of Canine and Human Lymphoma. Vet Sci. 2016;3(2):11. doi:10.3390/vetsci3020011.

13. Elisabeth M, Liebler-Tenorio RP. MALT structure and function in farm animals. Vet Res. 2006;37:257-280. doi:10.1051/vetres.

14. Song J, Sun D, Hong Y, Park G, Kim Y. MALT lymphoma at the base of tongue of a 29year-old woman treated with radiation therapy alone. J Cancer Res Ther. 2014;10(2):407409. doi:10.4103/0973-1482.136673.

This article is protected by copyright. All rights reserved. 
15. Sakabe H, Bamba M, Nomura K, et al. MALT Lymphoma at the Base of the Tongue Developing without any Background of Immunodeficiency or Autoimmune Disease. Leuk Lymphoma. 2003;44(5):875-878. doi:10.1080/1042819031000063390.

16. Goteri G, Ascani G, Filosa A, Corrado Rubini C, Olay S, Balercia P. Linfoma malt primario de la lengua. Prim malt limphoma tongue. 2004;9(5):459-463.

17. Lim J, Lim J-Y, Kim Y, et al. Primary diffuse large B cell lymphoma of the base of tongue. J Cancer Res Ther. 2012;8(1):135-137. doi:10.4103/0973-1482.95195.

18. Dennis MM, Ehrhart N, Duncan CG, Barnes AB, Ehrhart EJ. Lingual Lesions in Dogs / : 1,196 Cases (1995-2004). J Am Vet Med Assoc. 2006;228(10):1533-1537. doi:10.2460/javma.228.10.1533.

19. Syrcle J a, Bonczynski JJ, Monette S, Bergman PJ. Retrospective evaluation of lingual tumors in 42 dogs: 1999-2005. J Am Anim Hosp Assoc. 2008;44(6):308-319. doi:44/6/308 [pii]. 


\section{University Library}

\section{- M M N E R VA A gateway to Melbourne's research publications}

Minerva Access is the Institutional Repository of The University of Melbourne

\section{Author/s:}

Harris, LJ;Rout, ED;Hughes, KL;Labadie, JD;Boostrom, B;Yoshimoto, JA;Cannon, CM;Avery, PR;Ehrhart, EJ;Avery, AC

Title:

Clinicopathologic features of lingual canine T-zone lymphoma

\section{Date:}

2018-03-01

\section{Citation:}

Harris, L. J., Rout, E. D., Hughes, K. L., Labadie, J. D., Boostrom, B., Yoshimoto, J. A., Cannon, C. M., Avery, P. R., Ehrhart, E. J. \& Avery, A. C. (2018). Clinicopathologic features of lingual canine T-zone lymphoma. VETERINARY AND COMPARATIVE ONCOLOGY, 16 (1), pp.131-139. https://doi.org/10.1111/vco.12322.

Persistent Link:

http://hdl.handle.net/11343/293231 